\title{
Permanent Magnet Designs with Large Variations in Field Strength
}

\author{
Ramesh Gupta, Brookhaven National Laboratory, Upton, NY 11973 USA
}

\begin{abstract}
The use of permanent magnets has been investigated as an option for electron cooling ring for the proposed luminosity upgrade of RHIC. Several methods have been developed that allow a large variation in field strength. These design concepts were verified with computer simulations using finite element codes. It will be shown that the field uniformity is maintained while the field strength is mechanically adjusted.
\end{abstract}

Index Terms- Accelerator, Electron Cooling, Permanent Magnets.

\section{INTRODUCTION}

$\mathrm{T}$ HE proposed luminosity upgrade [1] of the Relativistic Heavy Ion Collider (RHIC) requires an electron cooling ring in a lattice that uses relatively low field magnets [2]. In the initial stages of this project, the use of permanent magnets was examined as a means to achieve low cost construction and operation. The required maximum pole tip field for cooling protons with an electron beam was either $0.14 \mathrm{~T}$ (low field design) or $0.4 \mathrm{~T}$ (medium field design) depending on the lattice chosen. Moreover, the required field for cooling heavy ions with an electron beam in any lattice is lower by a factor as large as $\mathbf{2 . 5}$. Therefore, these permanent magnets must have a mechanism for reducing field strength by a factor of 2.5 .

The relative field error in these magnets is typically kept below $0.1 \%$ from $x=-50 \mathrm{~mm}$ to $x=+50 \mathrm{~mm}$. It may be pointed out that in these low field magnets, better field quality can be obtained by shaping the iron pole, etc., if desired. It was found that the basic requirements could be met with the surplus ferrite blocks from the Fermilab's "Recycler Ring" and "8 GeV Beam Transport Line" projects $[3,4]$. Variable strength permanent magnets were also investigated for SSC [5].

A number of techniques have been investigated and developed that allow the field strength to be changed with the help of mechanical adjustments. This is done by providing a low reluctance path (shunt) to field lines that normally would go through the magnet aperture. The shunt is made of low carbon steel. These methods are capable of making changes much larger than the factor of 2.5 that is required for the ecooling project. In addition, magnet designs will be presented

Manuscript reccived October 20, 2003. This work was by U.S. Department of Encrgy under contract number DE-AC02-98CH10886.

The author is associated with the Brookhaven National Laboratory, Upton, NY I 1973 USA. Phonc: 1-631-344-4805, FAX: 1-631-344-2190 and Email: gupta@bnl.gov. that produce pole tip fields higher than the magnetization generated by the ferrite blocks.

\section{LOW FIELD DESIGN}

An OPERA 2-d [6] model of the magnet cross section is shown in Fig. 1. It has a horizontal aperture of $180 \mathrm{~mm}$ and vertical aperture of $31.75 \mathrm{~mm}$. Good field quality must be maintained in the $100 \mathrm{~mm}$ horizontal aperture $(50 \mathrm{~mm}$ half aperture). The outer yoke cross section is $240 \mathrm{~mm} \mathrm{X} 184 \mathrm{~mm}$. The field is generated by Strontium Ferrite bricks that were used in the Recycler project $\left(B_{r} \sim 0.38 T, H_{c} \sim 0.35 T\right)$. Typical dimension of these bricks are: $25.4 \mathrm{~mm}$ X $101.6 \mathrm{~mm}$ X 152.4 $\mathrm{mm}$. Two of these bricks are placed between the iron pole and iron yoke giving a total cross-section of $152.4 \mathrm{~mm} \mathrm{X} 50.8$ $\mathrm{mm}$. In this design, the iron pole shapes the field quality. The width of the yoke and of height of the iron pole are $12.7 \mathrm{~mm}$. The magnet has been designed to generate a maximum field of $\sim 0.21 \mathrm{~T}(50 \%$ higher than the design requirement of $0.14 \mathrm{~T})$ along with the capability to mechanically adjust the field by at least a factor of 5 .

$\gamma[\mathrm{mm}]$

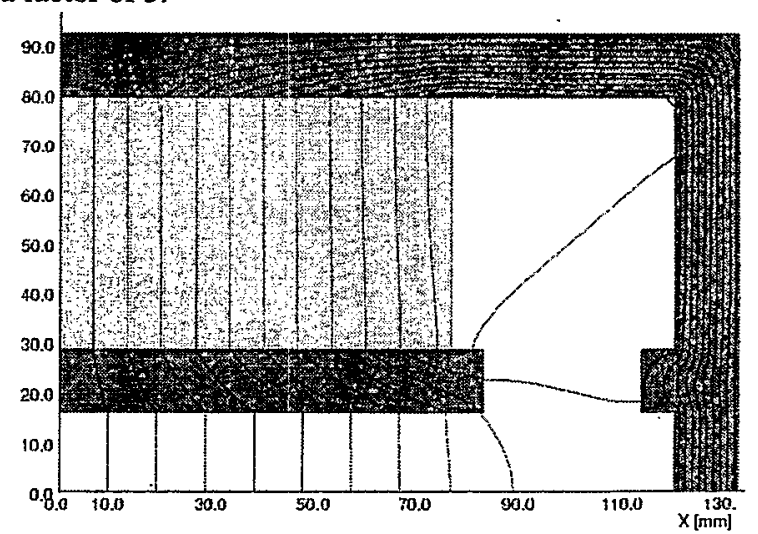

Fig 1: A quadrant of the magnct cross-scction that gencrates a nominal ficld of $0.21 \mathrm{~T}$. The ficld lines are superimposed on the magnet cross scetion. The ficld can be reduced with the help of iron shunt (between iron pole and return yoke) that is inserted from the right side.

\section{MEDIUM FIELD DESIGN}

A higher field (more than the residual field of Strontium Ferrite) can be generated by concentrating the flux lines in to a smaller iron pole. Two such designs are presented in Fig. 2 and Fig. 3. In both cases, the vertical aperture is $31.75 \mathrm{~mm}$ (same as in Fig 1) and the good field horizontal aperture is $100 \mathrm{~mm}$. The two create a central field of $\sim 0.4225 \mathrm{~T}$ and $\sim 0.4161 \mathrm{~T}$ respectively and they differ only in the way the 
pole is shaped to generate a higher field. The total crosssection of the Strontium Ferrite bricks in each half is 406.4 $\operatorname{mm} X 101.6 \mathrm{~mm}$. The outer dimensions of yoke are $646 \mathrm{~mm}$ X $406 \mathrm{~mm}$.

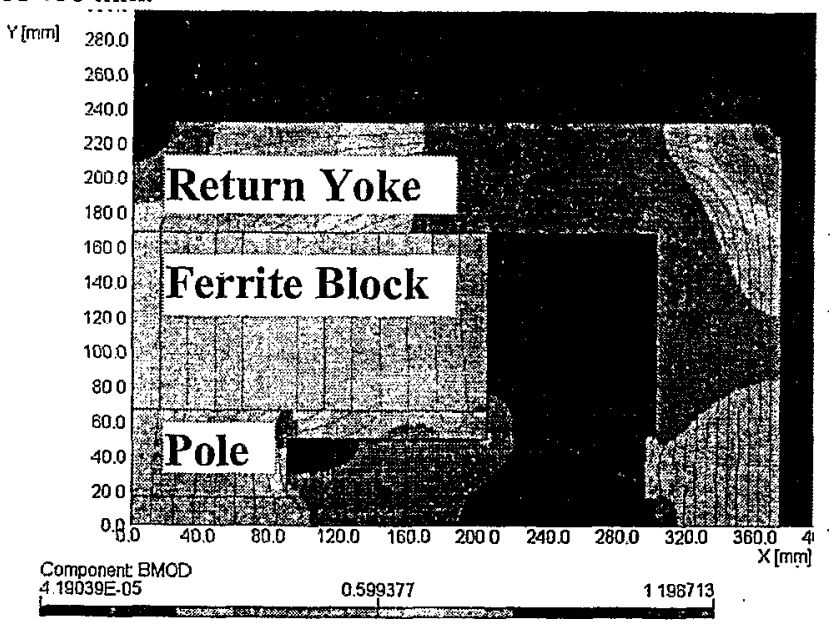

Fig 2: A quadrant of the magnct cross-scction that generates a nominal ficld of $\sim 0.423 \mathrm{~T}$. The magnitude of the ficld and the ficld lines are superimposed on the magnet cross section.

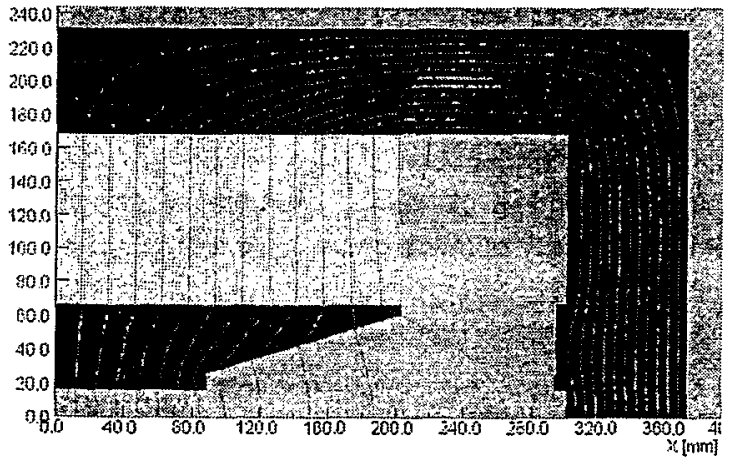

Fig. 3: A quadrant of the magnet cross-scetion that generates a nominal ficld of $-0.416 \mathrm{~T}$. The ficld lines are superimposed on the magnet cross section.

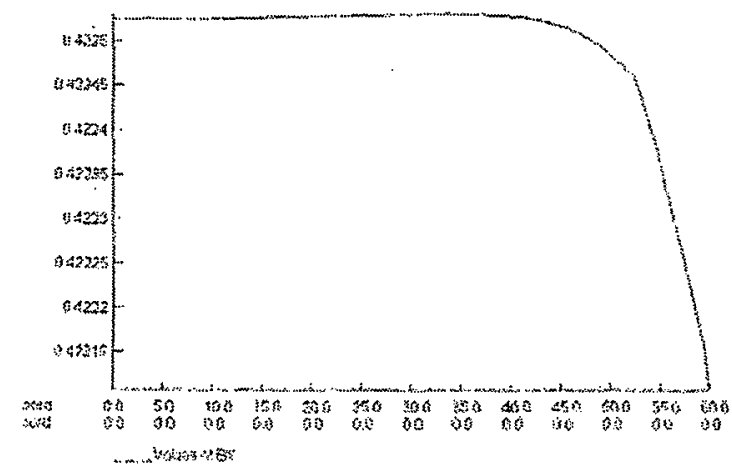

Fig. 4: The vertical ficld (By) on the horizontal axis in the design shown in Fig. 2. The maximum ficld is $\sim 0.4225 \mathrm{~T}$.

The variation of the field along the horizontal axis for the design shown in Fig 2 is shown in Fig. 4 and for the design shown in Fig. 3 is shown in Fig. 5. One can see that field uniformity of about a part in 1,000 is obtained in both cases.

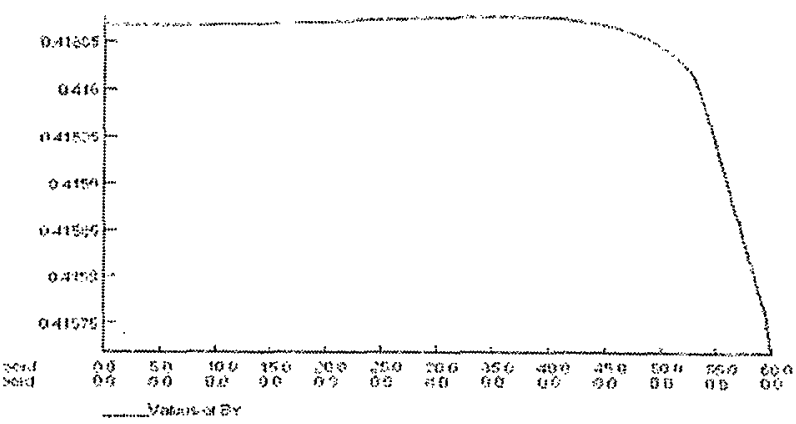

Fig. 5: The vertical ficld (By) on the horizontal axis in the design shown in Fig. 3. The maximum ficld is $\sim 0.4161 \mathrm{~T}$.

\section{ADJUSTMENT IN THE FIELD STRENGTH BY VARYING THE AMOUNT OF SHUNT IN THE MAGNET CROSS-SECTION}

In this section those techniques are discussed where the cross section of the shunt remains the same across the length of a long magnet. It is assumed that either the end effects can be neglected or the exact amount of shunt is adjusted using 3d calculations.

The shunt iron, which provides a bypass path to the part of field lines away from magnet aperture, is placed between the iron pole and return yoke. It can be done two ways, as shown in Fig. 6 and Fig. 7 (compare them to Fig. 1). In first method (Shunt Type A), the shunt iron, which has the same height as the pole, is partially inserted. A change in field strength is achieved by varying the amount of insert (a larger insert gives a larger reduction). In the second method (Shunt Type B), the shunt is always fully inserted but the height is changed to adjust the amount of field reduction needed. In this case, the shunt iron becomes well saturated. In Fig. 8, we show the field along the $X$-axis in the three cases: Original Case (see model in Fig. 1), Shunt Type A (see model in Fig. 6) and Shunt Type $B$ (see model in Fig. 7). In Fig. 9, we show the results of many simulations, where the amount of the shunt was adjusted to obtain different values of the field in the aperture. One can see that a reasonable field quality is maintained in all cases.

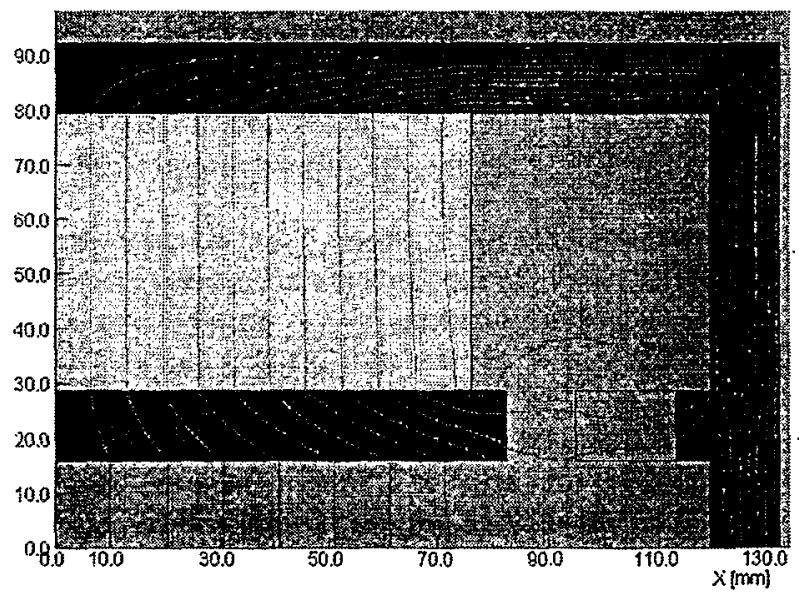

Fig. 6: Magnetic model of a quarter of the magnet in the method where a shunt of full height is partially inserted (Shunt Type A). 


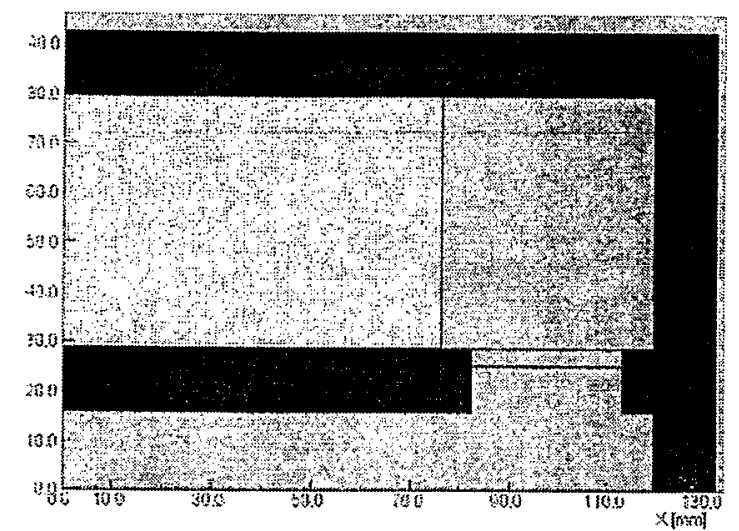

Fig. 7: Magnetic model of a quarter of the magnet in the method where a shunt of partial height is fully inscrted (Shunt Type B).

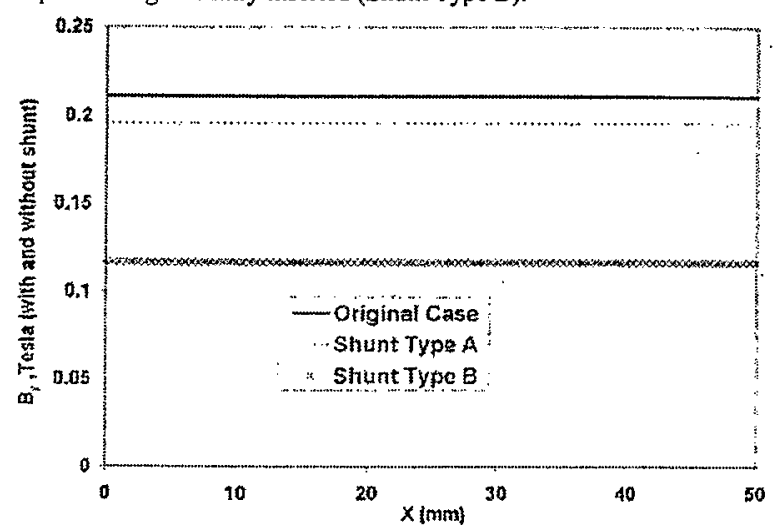

Fig 8: Ficld along the $X$-axis without shunt (Fig. 1) and with two types of shunts (Fig. 6 and Fig. 7). The shunt can be adjusted to change the ficld.

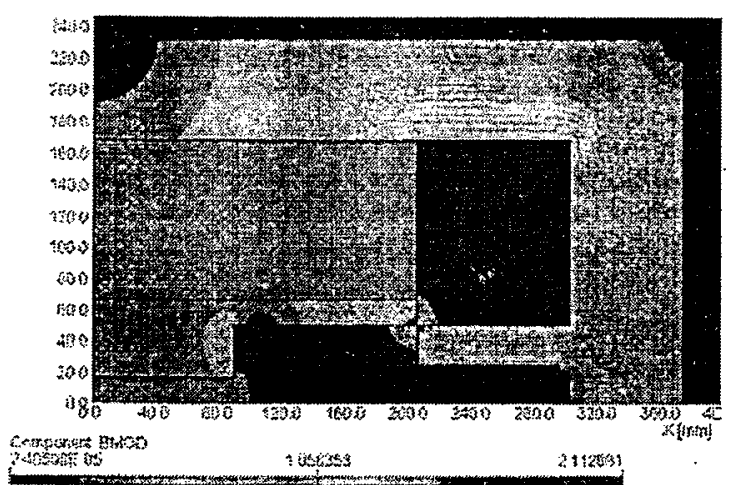

Fig. 9: Magnetic model of a quarter of the medium ficld magnet with a shunt that reduces the ficld in the magnet aperture from $0.42 \mathrm{~T}$ to $0.33 \mathrm{~T}$. A decper insertion of the shunt will give a larger reduction in ficld.

In Fig. 9, we present a shunt for the medium field magnet (see original model in Fig. 2). The shunt reduces the vertical field on the $\mathrm{X}$-axis from $0.42 \mathrm{~T}$ to $0.33 \mathrm{~T}$. A deeper insertion of this shunt will bring a larger reduction in the field.

\section{ADJUSTMENT IN THE FIELD STRENGTH BY VARYING THE AMOUNT OF SHUNT IN THE MAGNET LENGTH}

Another way of changing field strength is by changing the length of a shunt that occupies only part of the magnet length. The reduction in field strength is computed by $3-d$ codes such as OPERA 3-d. A simple example of such a shunt is a variation of the technique presented in the last section. In this technique, one uses a full width but partial length bar (shunt) where an iron bar connects/extends the pole to the return yoke and is placed at one or more location along the magnet length. The total length of the shunt determines the reduction in field.

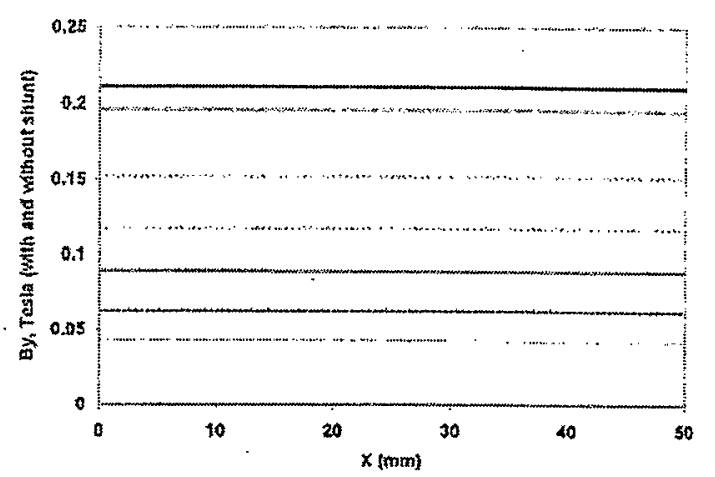

Fig 10: Ficld along the $X$-axis without shunt (top linc, model of Fig. 1). and with various shunts. This figure shows that the magnitude of the ficld can bc varied by a significant amount without producing a large distortion in the ficld.

The 3-d models analyzed in this section have the same cross section as those of models discussed in the previous section. Fig. 11 shows a computer model of one octant (one quadrant, half length) of the magnet without a shunt (see Fig. 1 for cross section) and Fig. 12 with shunt. In these models, the pole piece goes from $\mathrm{z}=0 \mathrm{~mm}$ to $\mathrm{z}=240 \mathrm{~mm}$. In Fig. 12, a 20 mm long shunt is placed starting at $z=200 \mathrm{~mm}$. Because of symmetry these models represent a total pole length of 480 $\mathrm{mm}(-240 \mathrm{~mm}$ to $+240 \mathrm{~mm})$ and the two shunts are placed symmetrically on either end of the magnet. The field strength along the $\mathrm{z}$-axis is plotted in Fig. 13 for the model shown in Fig. 11 (original case, no shunt, $0.2097 \mathrm{~T}$ maximum field) and Fig. 14 for the model shown in Fig. 12 (case with $20 \mathrm{~mm}$ long shunt). There is practically no relative variation in the field strength along the z-axis. As explained earlier, the iron shunt bypasses the flux lines away from magnet aperture, which, in turn, reduces the field in the aperture.

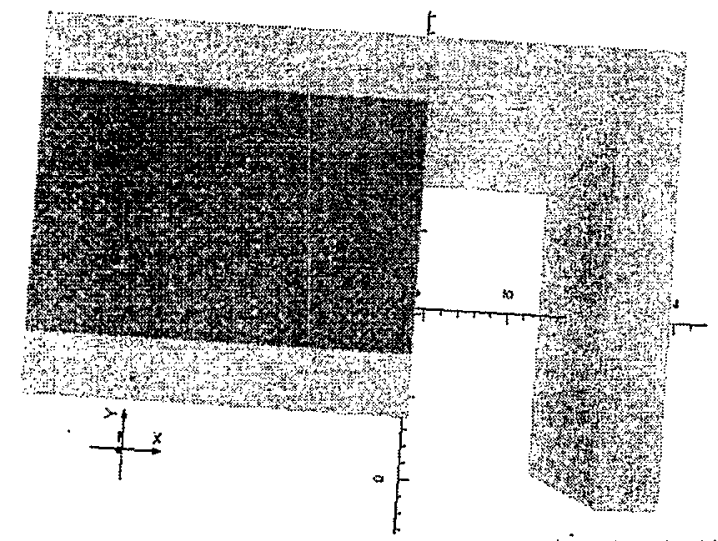

Fig 11: OPERA 3-d model of an octant the magnet (half length, 1/4 cross scction) that gencrates a nominal ficld of $0.21 \mathrm{~T}$ and does not have a shunt for reducing field. 
Another method of reducing the field is by inserting iron shunt plates in the same cross sectional space where normally the Strontium ferrite bricks are placed. To incorporate this scheme would leave spaces between the ferrite. The total thickness of shunt plates will determine the amount of field reduction (shunt). One such case is shown in Fig. 15 where a $20 \mathrm{~mm}$ thick shunt plate is put at the end of the magnet. The field reduction (from $0.2097 \mathrm{~T}$ to $0.1623 \mathrm{~T}$ ) along the $\mathrm{z}$-axis is shown in Fig. 16. The integral field in the magnet halves of the three models analyzed here (Fig. 11, Fig. 12 and Fig. 15) is respectively, 53.74 T.mm (no shunt), 49.29 T.mm (shunt bar) and 36.18 T.mm (shunt plate), as indicated at the bottom left of Figs. 13, 14 and 16.

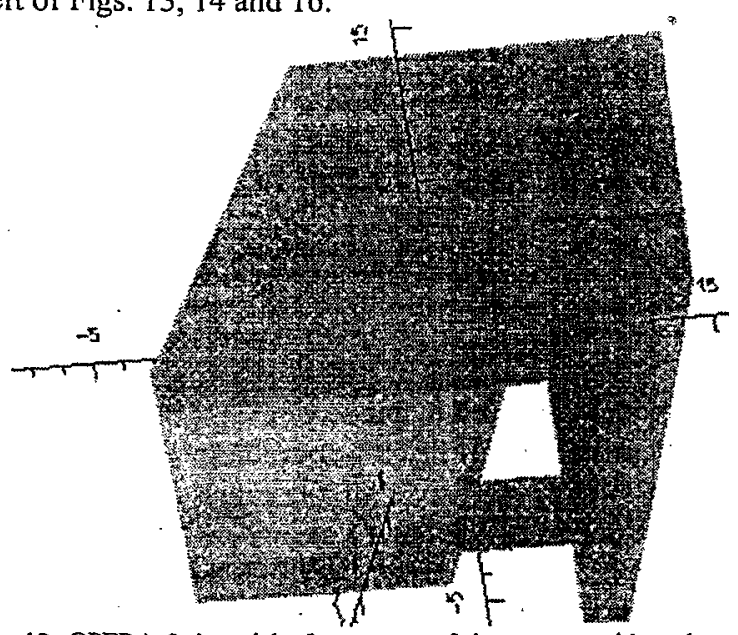

Fig 12: OPERA 3-d model of an octant of the magnet with a shunt bar of partial length between the pole and the return yoke for reducing the ficld.

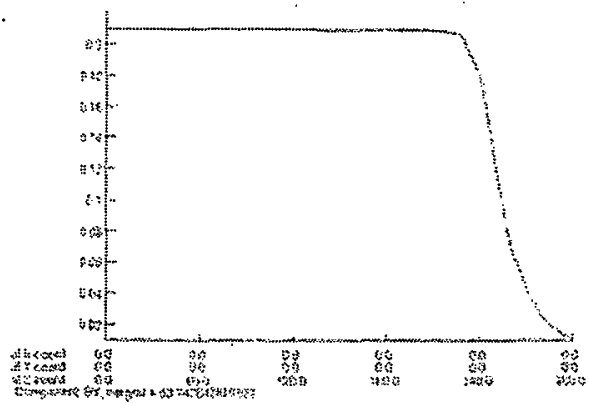

Fig 13: The ficld along the z-axis in the abscnce of any shunt (model shown in Fig. 11). The maximum ficld is $\sim 0.2097 \mathrm{~T}$.

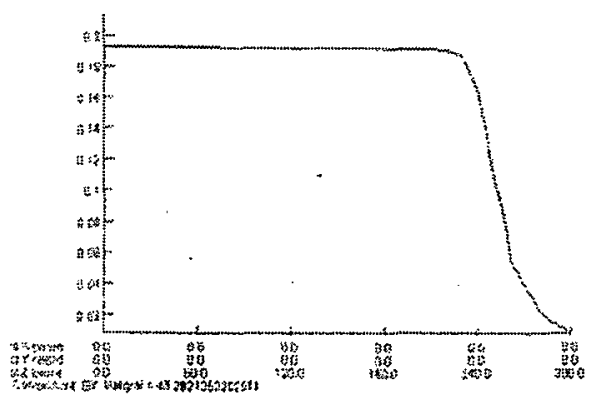

Fig 14: The ficld along the z-axis with a shunt bar of partial length for reducing ficld (model shown in Fig. 12). The maximum field is $\sim 0.1928 \mathrm{~T}$.

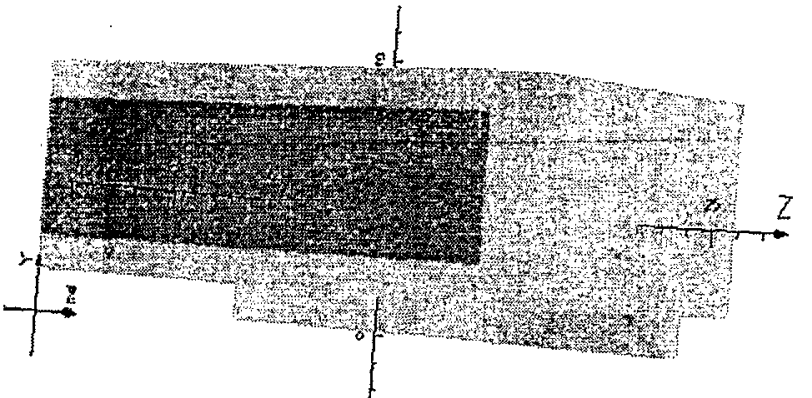

Fig 15: OPERA 3-d model of an octant of the magnet with a shunt plat at end of the magnet reducing ficld.

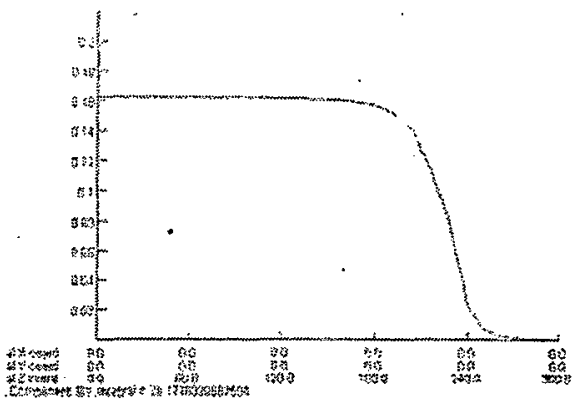

Fig 16: The ficld along the z-axis with a shunt plate at either end for reducing ficld (model shown in Fig. 15). The maximum ficld is $\sim 0.1623 \mathrm{~T}$.

\section{SUMMARY}

A number of techniques for adjusting (reducing) field in permanent magnets have been presented. These techniques rely on an adjustable shunt that provides a low reluctance path to bypasses field lines from the aperture of a magnet to the return yoke. Designs are also investigated where the field in the aperture can be increased beyond the residual field of the magnetized bricks by concentrating flux lines at the iron pole.

\section{ACKNOWLEDGMENT}

We appreciate discussions with M. Anerella, I. Ben-zvi, M. Harrison, J. Kewish and S. Plate. We thank W. Foster and J. Volk of Fermilab for their valuable help on various topics related to permanent magnets and for coordinating potential use of surplus ferrite bricks in this project. We thank P. Wanderer for his constructive comments on the manuscript of this paper.

\section{REFERENCES}

[1] I. Ben-Zvi, et al., "R\&D Towards Cooling of the RHIC Collider," Particle Accelcrator Confercnce, Portland, Orcgon, May 12-16, 2003.

[2] J. Kcwish, ct al., "Layout and Optics for RHIC Electron Cooler," Particlc Accclerator Conference, Portland, Oregon, May 12-16, 2003.

[3] W. Foster, ct al., "Expcrience with Permanent Magnets in the Fermilab 8 GeV Linc and Recycler Ring", $6^{\text {th }}$ European Particle Accelerator Confercnce, Stockholm, Junc 22-26, 1998.

[4] J. Volk, ct al., "Time Evolution of Ficlds in Strontium Ferrite Permanent Magncts," Particle Accclcrator Conference, Chicago, June 18-22, 2001.

[5] OPERA 2-d and OPERA 3-d are among a sct of finitc clement computer codes from Vector Ficlds, Inc. hittp://www.vectorficlds.com.

[6] D.B. Barlow, ct al., "Variable Ficld Permanent Magnet Quadrupole for the SSC", IEE Trans. of Magnctics,'Vol. 30, No. 4, pp 2288-229I. 